\title{
Torque Coordination Control During Mode Transition for a Series-Parallel Hybrid Electric Vehicle
}

\author{
Li Chen, Gang Xi, and Jing Sun, Fellow, IEEE
}

\begin{abstract}
Mode transitions are significant events in the operation of series-parallel hybrid electric vehicles (SPHEVs) with a clutch serving as the key enabling actuator element. Due to the friction-induced discontinuity of the clutch torque, seamless transition is difficult to achieve. In this paper, a model reference control (MRC) law is proposed to coordinate the motor torque, engine torque, and clutch torque to manage transitions. The control system is overactuated in the sense that three inputs (i.e., three torques) can be manipulated to control the two outputs (angular speeds of the two sides of the clutch). The effects of using different input combinations are analyzed to exploit the overactuation feature of the system, and performance sensitivities to various design factors are studied. The simulation and experimental results from an SPHEV bus demonstrate that the MRC achieves reduced torque interruption, less vehicle jerk, and smaller frictional losses, compared to the conventional operation method.
\end{abstract}

Index Terms-Clutch engagement, hybrid electric vehicle (HEV), mode transition, model reference control (MRC).

\section{NOMENCLATURE}

$A \quad$ Friction area of each friction face.

$A_{V}$

$c_{D}$

$d_{1}, d_{2}$

$e_{1}, e_{2}$

$f$

$g$

$i_{0}$

$i_{1}$

$I_{\mathrm{tm}}$

$J_{1}$

$J_{2}$

$J_{\text {cltch }}$
$J_{\text {eng }}$
$J_{\text {fnl_gear }}$
$J_{\text {fst_gear }}$
$J_{\text {isg }}$
$J_{\text {tm }}$
$k_{i j}(i=1,2,3)$
$\quad(j=1,2)$
$m$
$N$
$P_{c}$
$P_{c \_m a x}$
$r$
$r_{i}(i=1,2,3)$
$R$
$R_{c}$
$T_{c}$
$T_{e}$
$T_{f}$
$T_{\text {tm }}$
$T_{\text {tm }}$
$T_{\text {tm_cmd }}$

Manuscript received August 8, 2011; revised February 4, 2012; accepted April 15, 2012. Date of publication May 21, 2012; date of current version September 11, 2012. This work was supported by the Chinese National Science Foundation under Grant 51105244. The review of this paper was coordinated by Dr. A. Davoudi.

L. Chen is with the School of Mechanical Engineering, Shanghai Jiao Tong University, Shanghai 200240, China, and also with the National Laboratory of Automotive Electronics and Control, Shanghai 200240, China (e-mail: li.h.chen@sjtu.edu.cn).

G. Xi is with the United Automotive Electronic Systems Company, Ltd., Shanghai 201206, China (e-mail: gang.xi@uaes.com).

J. Sun is with the Department of Naval Architecture and Marine Engineering, University of Michigan, Ann Arbor, MI 48109 USA (e-mail: jingsun@ umich.edu).

Color versions of one or more of the figures in this paper are available online at http://ieeexplore.iee.org.

Digital Object Identifier 10.1109/TVT.2012.2200305
Moment of inertia of the clutch frictional plates.

Moment of inertia of the engine.

Moment of inertia of the final gear.

Moment of inertia of the first gear.

Moment of inertia of the integrated starter and generator.

Moment of inertia of the traction motor.

Feedback gains.

Vehicle mass.

Number of friction faces.

Normal pressure on the clutch plate.

Maximum normal pressure.

Command input.

Decomposed reference inputs.

Tire radius.

Equivalent acting radius of the friction torque on the clutch plate.

Clutch torque.

Engine torque.

Resistant torque due to friction and air compression of the engine.

Traction motor torque.

Equivalent traction motor torque with respect to Shaft B.

Vehicle load torque.

Equivalent vehicle load torque with respect to Shaft B.

Desired traction torque.

Equivalent desired traction torque with respect to Shaft B.

$u_{i}(i=1,2,3) \quad$ Control inputs.

$v \quad$ Vehicle speed.

$v_{\text {rel }} \quad$ Relative wind velocity in vehicle running direction.

Head wind speed.

State variables of the plant.

State variables of the reference model.

Outputs of the plant.

Output of the reference model.

Road inclination angle.

Slipping friction coefficient of the clutch.

Static friction coefficient of the clutch.

Air density. 

$\omega_{1}$
$\omega_{2}$
$\omega_{\mathrm{tm}}$
$\omega_{m}$

\begin{abstract}
Angular speed of Shaft A.
Angular speed of Shaft B.

Traction motor speed.

Desired speed of the reference model.
\end{abstract}

\section{INTRODUCTION}

$\mathbf{S}$ ERIES-PARALLEL hybrid electric vehicles (SPHEVs, which are also referred to as split-power or complexhybrid) have multiple power paths and can be configured to run in both series and parallel modes [1]. By leveraging the special features of both modes, SPHEV s have the potential to improve fuel economies and significantly reduce carbon emissions [2], [3]. To make full use of the SPHEV powertrain topology, frequent transitions between different modes are necessary to optimize vehicle operation [4]-[6]. Some of these transitions, if improperly managed, might negatively impact noise vibration and harshness performance, resulting in negative customer perception on drivability performance. Therefore, handling the transitions to achieve seamless mode switching is a critical control task for SPHEV development.

Various hardware mechanisms have been proposed for integrating series and parallel power flows for SPHEVs, including planetary gears [7]-[9], continuous variable transmissions [10], and switchable powertrains using clutches [6], [11], [12]. Among these available technologies, clutches are excellent choices as SPHEV power-switching device due to their high efficiency and compact size, and the maturity of the technology [2], [11].

A clutch transfers the power source from one driving device to another for the hybrid electric vehicle (HEV) powertrain using friction torques. At any given time, the clutch can be in one of three phases of the engagement process: 1) open; 2) slipping; and 3) locked. The open phase of a clutch is trivialthe two sides are not connected. The clutch is in the slipping phase when the two sides are in contact, but their angular speeds are not synchronized yet. In the slipping phase, the angular speeds of the two sides are different, i.e., there are two degrees of freedom (DoFs) in the powertrain rotational dynamics. Moreover, the dynamics of the two DoFs are driven by three input torques from the motor, engine, and clutch, respectively. Therefore, the number of control inputs is one more than the number of control DoFs, making the clutch-coupled powertrain in the slipping phase an overactuated system. To reach the desired angular speeds of the two sides, multiple theoretical solutions exist using different combinations of the inputs. Developing a systematic method for selecting a proper combination of the inputs for this overactuated system under various operating constraints and formulating the criterion to coordinate these inputs are two of the primary interests pursued in this paper.

One special challenge in managing mode transition for the clutch-enabled SPHEV is the switch from the slipping phase to the locked phase (slip-stick transition), as the clutch friction torque introduces nonlinear dynamics to the powertrain and makes the clutch operation very complicated. While the friction torque in the slipping phase is proportional to the clutch pressure, which can be controlled by its actuator, the friction torque in the locked phase can assume any value, up to an upper limit determined by the clutch pressure [13]. Thus, if the slipping process is not carefully controlled, the friction torque may become discontinuous and abruptly change when the slipstick transition takes place. This discontinuity may result in an intensive vehicle jerk and lead to unfavorable customer drivability perception.

To address this problem, model predictive control approaches have been applied to regulate the torques of the motor and the clutch [14]. A subdomain controller based on state space partitions was proposed for a class of switchable hybrid dynamical systems, and a control method with control inputs composed of a feedforward linearizing component and a linear feedback component was developed for engine start-stop switching operation [4]. In this work, the clutch pressure was not considered as a control variable and was treated as a known disturbance. However, this approach has its limits when dealing with nonlinearities associated with the clutch engagement dynamics. A control sequence and two clutch slipping control methods using the slipping speed were introduced for mode transition in [5]. Fast disengaging and engaging strategies were used to deliver continuous power for transmission-actuated HEVs [15], [16]. As the whole, existing solutions found in literature are based on heuristic techniques and do not emphasize the modeling aspect or explicitly include the clutch model in their control designs. A model-based approach would provide a systematic design framework, reveal more operational insights, and may have the potential to achieve better results, thereby motivating the work reported in this paper.

This paper proposes a model reference control (MRC) to achieve smooth transitions with reduced driveline interruption and frictional losses for SPHEVs equipped with a clutch. A typical transition from motor-only mode to compound driving mode is analyzed in depth to gain insight into the vehicle dynamics. Given that the longitudinal dynamics of the vehicle are controlled by the traction motor torque solely in the motor-only driving mode but by the traction motor torque and engine torque in the compound driving mode, one key function of the mode transition is to infuse the engine torque into the powertrain by a controlled clutch torque during the transition phase. For a seamless mode transition where no disturbance is introduced to the vehicle dynamics, the vehicle is expected to run as if it were still in the motor-only driving mode. This desired feature can be refined into the following control design objective: to coordinate the motor, engine, and clutch torques, so that the vehicle tracks the vehicle dynamics in the motor-only driving mode. This objective naturally renders the MRC as the design framework, for which the desired performance is expressed by a reference model that will be mimicked by the plant through feed-forward and feedback control, as shown in Fig. 1 [17]. Hence, the target output of the plant is the transient output of the reference model, instead of a constant or predefined value, which is impractical for a running vehicle. The MRC solutions have been well established for many nonlinear problems, such as the shunt activepower-filter system [18], the piezopositioning system [19], and the three-phase three-level boost rectifier [20]. Nevertheless, relevant literatures on overactuated systems with discontinuous problems have not been found, to the best of our knowledge. 


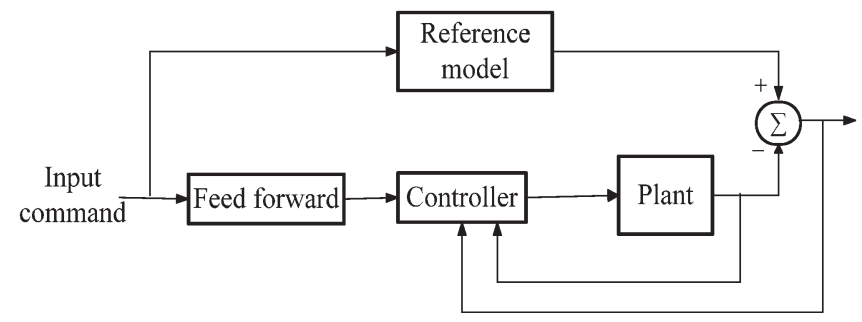

Fig. 1. MRC architecture.

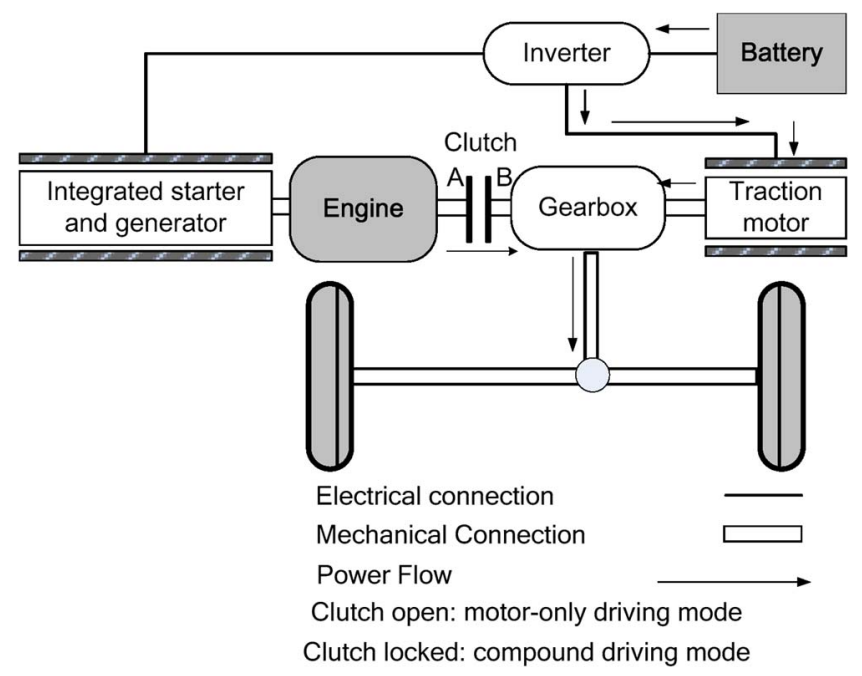

Fig. 2. Two of the operating modes of an SPHEV.

Under the MRC architecture, a reference model is built to capture the desired powertrain dynamics of the motor-only driving mode. An output feedback MRC algorithm is proposed, the conditions for closed-loop system stability are derived, and the methods for selecting input combinations and controller parameters are discussed. The MRC method is applied to an SPHEV bus. The simulation and experimental results presented establish the effectiveness of MRC and are compared to outcomes for a control system developed by a conventional method.

\section{System Description AND PROBlem Formulation}

Consider a posttransmission SPHEV shown in Fig. 2, in which an integrated starter and generator and a traction motor are added to the powertrain. Two of the common operating modes are shown: motor-only and compound driving. In the motor-only driving mode, the clutch is open, and the traction motor drives the vehicle using solely electric energy from the battery. In the compound driving mode, the clutch is locked, and the traction motor drives the vehicle, together with the engine. When the traction motor torque decreases to zero, the SPHEV enters into engine-only driving mode. Therefore, the mode transition from motor only to compound driving is also a critical and necessary process for the power source transition from motor-only to engine-only driving.

With proper energy management strategy, it has been estimated that this SPHEV with an engine of $110 \mathrm{~kW}$ (maximum), a traction motor of $40 \mathrm{~kW}$ (continuous), and an integrated starter and generator of $20 \mathrm{~kW}$ (continuous) can reduce theoretical fuel consumption by up to $30 \%$ in Chinese transit buses [12]. However, experiments have shown that the mode transition from motor only to compound driving may cause disturbances to the output torque, leading to jerky motions and excessive wear to the clutch friction plates. The mode shifting has been a concern in hybrid vehicles with similar configurations [21].

The mode transition from motor only to compound driving can be carried out in two steps. In the first step, the engine speed is increased, and the clutch is slipping and then locked; in the second step, the clutch is locked, the motor torque is reduced, and the engine torque is gradually increased. In the first step, the vehicle longitudinal dynamics are sensitive to the clutch torque profile during the clutch-slipping phase. However, in the second step, the clutch torque no longer affects the vehicle dynamics once the clutch is locked. Thus, the torque coordination problem is more challenging for the first step, which is the focus of this paper.

Although many researchers have studied dynamics and control during clutch engagement for conventional vehicles [22], [23], their strategies and conclusions are not directly applicable for the problem at hand, given the differences between SPHEVs and conventional vehicles. For SPHEVs, one more external torque, i.e., the motor torque, is applied to the powertrain, together with the engine torque. Accordingly, their control objectives are different. For conventional vehicles with the engine as the only power source, the primary objective during the clutch engagement is to quickly and smoothly connect the engine to the vehicle. However, for SPHEVs, the engine and the traction motor are two alternative power sources, and thus, the objective is to smoothly engage the clutch without causing torque interruption, regardless of which source is powering the vehicle.

\section{SPHEV DyNAMIC MODEL}

To facilitate the model-based design proposed in this paper, a control-oriented SPHEV model capturing the mode transition dynamics is first developed and presented. The vehicle driveline during an SPHEV mode transition can be viewed as a multibody system. To simplify the analysis, the damping and compliance of the clutch are ignored, and all parts of the clutch are assumed to be lumped inertias [22]. The driveline dynamic model is shown in Fig. 3(a), and its equivalent simplified model is built in Fig. 3(b). One side of the clutch is connected to the engine by Shaft A, and the other side is connected to the gearbox, traction motor, and vehicle body by Shaft B.

The dynamic equations in the mode transition of the SPHEV driveline can be written as

$$
\begin{aligned}
& J_{1} \dot{\omega}_{1}(t)=T_{e}(t)-T_{f}(t)-T_{c}(t) \\
& J_{2} \dot{\omega}_{2}(t)=\bar{T}_{\mathrm{tm}}(t)+T_{c}(t)-\bar{T}_{r}(t) .
\end{aligned}
$$

Equations (1) and (2) represent the dynamics of Shaft A and Shaft B, respectively. 


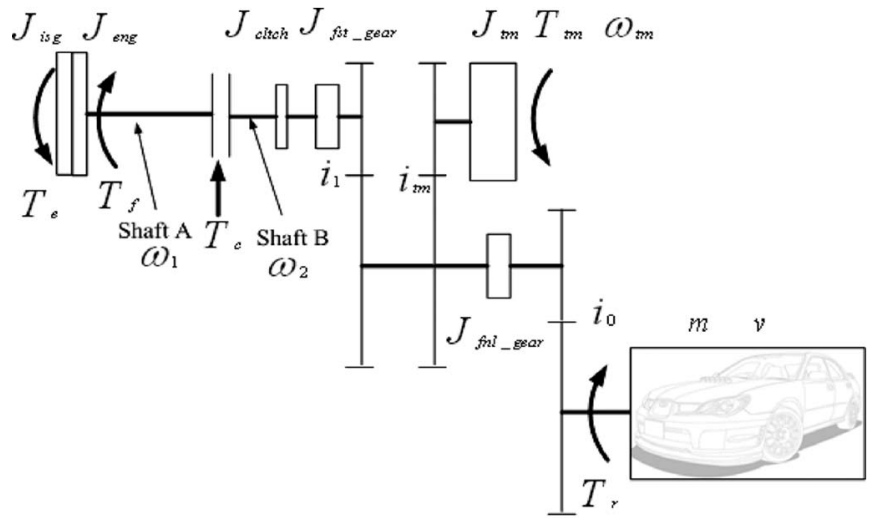

(a)

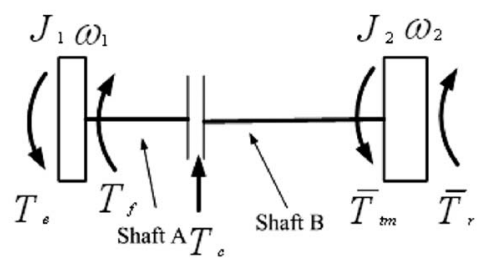

(b)

Fig. 3. Dynamic model of the SPHEV driveline. (a) Driveline model. (b) Equivalent model.

\section{A. Kinematic Deduction}

The calculation of $T_{r}$ is typically a composition of the rolling, gravity, and wind resistance. The relevant terms are expressed as [24]

$$
\begin{aligned}
F_{\text {air }} & =0.5 \cdot c_{D} \cdot A_{V} \cdot \rho_{\text {air }} \cdot v_{\text {rel }}^{2} \\
& =0.5 \cdot c_{D} \cdot A_{V} \cdot \rho_{\text {air }} \cdot\left(v_{\text {air }}+v\right)^{2} \\
F_{g} & =m \cdot g \cdot \sin \alpha \\
F_{r} & =m \cdot g \cdot \cos \alpha \cdot f \\
T_{r} & =\left(F_{\text {air }}+F_{g}+F_{r}\right) \cdot R .
\end{aligned}
$$

The calculations of $J_{1}, J_{2} \bar{T}_{\mathrm{tm}}$, and $\bar{T}_{r}$ are given by

$$
\begin{aligned}
J_{1}= & J_{\text {eng }}+J_{\text {isg }} \\
J_{2}= & \frac{m R^{2}}{i_{0}^{2} i_{1}^{2}}+\frac{J_{\mathrm{tm}}}{i_{1}^{2}} i_{\mathrm{tm}}^{2}+J_{\text {cltch }} \\
& +J_{\text {fst_gear }}+\frac{J_{\text {fnl_gear }}}{i_{1}^{2}} \\
\bar{T}_{\mathrm{tm}}= & \frac{T_{\mathrm{tm}} \cdot i_{\mathrm{tm}}}{i_{1}} \\
\bar{T}_{r}= & \frac{T_{r}}{i_{1} \cdot i_{0}} .
\end{aligned}
$$

The calculations of $v$ and $\omega_{\mathrm{tm}}$ from $\omega_{2}$ are given by the following equations:

$$
\begin{aligned}
\omega_{\mathrm{tm}} & =\omega_{2} \cdot \frac{i_{\mathrm{tm}}}{i_{0}} \\
v & =\omega_{2} \cdot \frac{R}{i_{0} \cdot i_{1}} .
\end{aligned}
$$

\section{B. Clutch Model}

Let $c$ represent the contact status of the two sides of the clutch, i.e., $c=1$ when the two sides are in contact with each other and $c=0$ when the two sides are separated. When $c=1$, the Coulomb friction model, which is calculated using a signum function $\operatorname{sign}(\cdot)$, is applied to $T_{c}$, i.e.,

$$
\begin{aligned}
& \text { when } c=1 \text { and } \omega_{1}(t) \neq \omega_{2}(t): \\
& T_{c}(t)=\mu_{s} \cdot P_{c}(t) \cdot R_{c} \cdot N \cdot A \cdot \operatorname{sign}\left(\omega_{1}(t)-\omega_{2}(t)\right) \\
& \text { when } c=1 \text { and } \omega_{1}(t)=\omega_{2}(t): \\
& T_{c}(t)=\left[-\mu_{l} \cdot P_{c}(t) \cdot R_{c} \cdot N \cdot A \quad \mu_{l} \cdot P_{c}(t) \cdot R_{c} \cdot N \cdot A\right] .
\end{aligned}
$$

For the locked phase, the range of $T_{c}$ is given by (14), but the value is not given. The calculation of $T_{c}$ in this case is derived from (1) and (2) for $\omega_{1}(t)-\omega_{2}(t)=0$ as

$$
\begin{aligned}
& \text { when } c=1 \text { and } \omega_{1}(t)=\omega_{2}(t): \\
& \begin{aligned}
T_{c}(t)= & \frac{J_{2}}{J_{1}+J_{2}} T_{e}(t)-\frac{J_{1}}{J_{1}+J_{2}} \bar{T}_{\mathrm{tm}}(t) \\
& -\frac{J_{2}}{J_{1}+J_{2}} T_{f}(t)+\frac{J_{1}}{J_{1}+J_{2}} \bar{T}_{r}(t)
\end{aligned}
\end{aligned}
$$

subject to :

$$
T_{c}(t) \in\left[-\mu_{l} \cdot P_{c} \cdot R_{c} \cdot N \cdot A \quad \mu_{l} \cdot P_{c} \cdot R_{c} \cdot N \cdot A\right] .
$$

For the open phase, no torque is transferred through the clutch, which means

$$
\text { when } c=0: T_{c}(t)=0 \text {. }
$$

\section{Reference Model}

The vehicle dynamics of the motor-only driving mode are considered as the reference model. In this mode, the vehicle is powered by a desired traction torque $T_{\text {cmd }}$ acting on the same shaft of the traction motor. The equivalent torque acting on Shaft B, which is denoted by $\bar{T}_{\text {tm_cmd }}$, is calculated from $T_{\text {cmd }}$ as

$$
\bar{T}_{\mathrm{tm} \_\mathrm{cmd}}=\frac{T_{\mathrm{cmd}} \cdot i_{\mathrm{tm}}}{i_{1}} .
$$

The clutch is assumed to be locked in the reference model as the clutch is indeed locked when the engagement is completed. Therefore, the desired angular speeds of Shaft A and Shaft B are identical, and consequently, the dynamic equation for the reference model is

$$
\left(J_{1}+J_{2}\right) \dot{\omega}_{m}(t)=\bar{T}_{\text {tm_cmd }}(t)-T_{f}(t)-\bar{T}_{r}(t) .
$$

\section{Model Reference Controller Design}

To proceed with the control design using MRC, we first streamline the notations and introduce the following state 


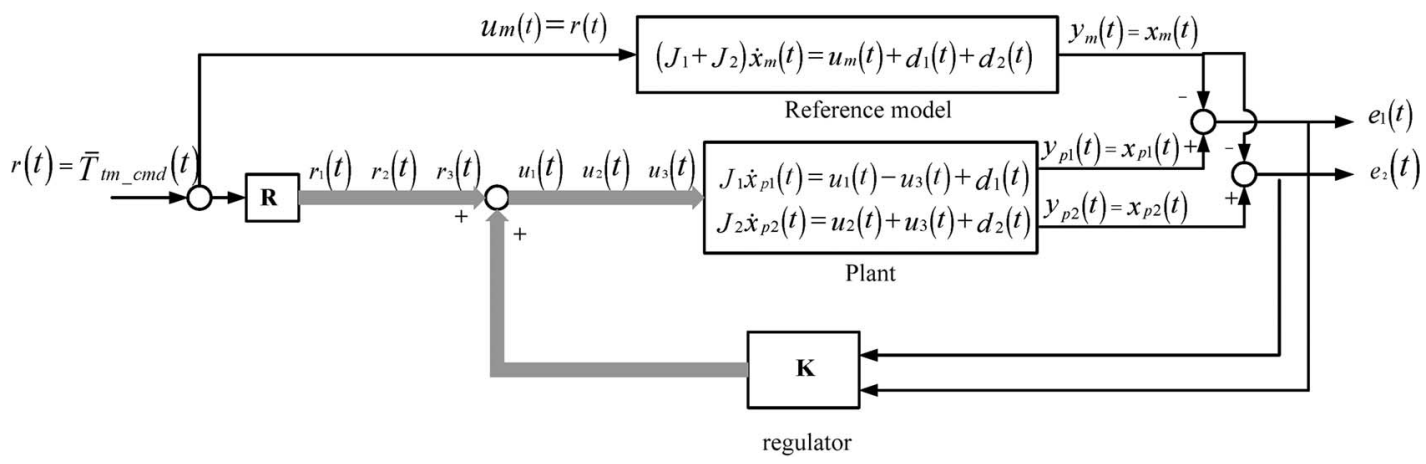

Fig. 4. Proposed MRC scheme.

variables:

$$
x_{p 1}(t)=\omega_{1}(t) \quad x_{p 2}(t)=\omega_{2}(t) .
$$

The inputs and outputs of the plant are denoted as

$$
\begin{aligned}
u_{1}(t) & =T_{e} \quad u_{2}(t)=\bar{T}_{\mathrm{tm}} \quad u_{3}(t)=T_{c} \\
y_{p 1}(t) & =x_{p 1}(t) \quad y_{p 2}(t)=x_{p 2}(t) .
\end{aligned}
$$

From (13)-(16), the normal pressure $P_{c}$ is calculated from $T_{c}$ as

$$
\begin{aligned}
& \text { when } c=1 \text { and } \omega_{1}(t) \neq \omega_{2}(t): \\
& \qquad P_{c}(t)=\operatorname{abs}\left(T_{c}(t)\right) /\left(\mu_{s} \cdot R_{c} \cdot N \cdot A\right) \\
& \text { when } c=1 \text { and } \omega_{1}(t)=\omega_{2}(t): \\
& \qquad P_{c}(t)=P_{c_{-} \max }
\end{aligned}
$$

when $c=0$ :

$$
P_{c}(t)=0 .
$$

External disturbances $\bar{T}_{r}(t)$ and $T_{f}(t)$ usually cannot be directly measured. However, we use estimated values for the controller derivation. The calculation of $\bar{T}_{r}(t)$ is given by (10), and $T_{f}(t)$ is estimated as a constant (whose estimation error and its effects are considered in Section V-B3). Here, the estimated values of $\bar{T}_{r}(t)$ and $T_{f}(t)$ are denoted by

$$
\begin{aligned}
& d_{1}(t)=-T_{f}(t) \\
& d_{2}(t)=-\bar{T}_{r}(t) .
\end{aligned}
$$

The state equations are then obtained by using the dynamic equations (1) and (2) as follows:

$$
\begin{aligned}
& J_{1} \dot{x}_{p 1}(t)=u_{1}(t)-u_{3}(t)+d_{1}(t) \\
& J_{2} \dot{x}_{p 2}(t)=u_{2}(t)+u_{3}(t)+d_{2}(t) .
\end{aligned}
$$

For the reference model, a dynamic equation can also be derived from (17) by assuming the state variable $x_{m}(t)=$ $\omega_{m}(t)$ and the input $u_{m}(t)=\bar{T}_{\mathrm{tm} \_ \text {cmd }}(t)$

$$
\left(J_{1}+J_{2}\right) \dot{x}_{m}(t)=u_{m}(t)+d_{1}(t)+d_{2}(t)
$$

with the output of the reference model being

$$
y_{m}(t)=x_{m}(t) .
$$

The error vector between the reference model and the plant is defined as

$$
\mathbf{e}(t)=\left(\begin{array}{l}
e_{1}(t) \\
e_{2}(t)
\end{array}\right)=\left(\begin{array}{l}
y_{p 1}(t)-y_{m}(t) \\
y_{p 2}(t)-y_{m}(t)
\end{array}\right) .
$$

The control goal is to achieve $\mathbf{e}(t)=0$. An output feedback control is proposed as in Fig. 4, where the thin lines represent scalar signals and the thick lines represent vector signals. $r(t)$ is a command input to the control system, which represents the desired traction torque $\bar{T}_{\mathrm{tm} \_ \text {cmd }}$ of the vehicle. $\mathbf{R}$ is a feed-forward regulator, which generates the reference inputs $r_{1}(t), r_{2}(t)$, and $r_{3}(t)$. $\mathbf{K}$ represents the gain for the feedback regulator. $e_{1}(t)$ and $e_{2}(t)$ are two outputs of interest from the closed-loop system.

The proposed output feedback control algorithms are given as follows:

$$
\begin{aligned}
& u_{1}(t)=r_{1}(t)+k_{11} e_{1}(t)+k_{12} e_{2}(t) \\
& u_{2}(t)=r_{2}(t)+k_{21} e_{1}(t)+k_{22} e_{2}(t) \\
& u_{3}(t)=r_{3}(t)+k_{31} e_{1}(t)+k_{32} e_{2}(t)
\end{aligned}
$$

where the feedback gains $k_{i j}(i=1,2,3 j=1,2)$ will be selected to meet the control objectives. The input to the reference model is

$$
u_{m}(t)=r(t)=\bar{T}_{\text {tm_cmd }}(t) .
$$

\section{A. Error Equations and Stability Conditions}

The choice of the feedback gain in (28)-(30) is subject to many constraints including the stability conditions. In the sequel, we first derive the stability conditions; then, we consider other design specifications that will impose further constraints on the selection. From (31), the time derivative of the error vector is written as

$$
\left(\begin{array}{c}
\dot{e}_{1}(t) \\
\dot{e}_{2}(t)
\end{array}\right)=\left(\begin{array}{c}
\dot{y}_{p 1}(t)-\dot{y}_{m}(t) \\
\dot{y}_{p 2}(t)-\dot{y}_{m}(t)
\end{array}\right)=\left(\begin{array}{c}
\dot{x}_{p 1}(t)-\dot{x}_{m}(t) \\
\dot{x}_{p 2}(t)-\dot{x}_{m}(t)
\end{array}\right) .
$$

To derive the error dynamics, we use (27)-(29) and (32)-(34) that yield (37), shown at the bottom of the next page, where

$$
\mathbf{A}=\left(\begin{array}{ll}
\frac{k_{11}-k_{31}}{J_{1}} & \frac{k_{21}-k_{32}}{J_{1}} \\
\frac{k_{21}+k_{31}}{J_{2}} & \frac{k_{22}+k_{32}}{J_{2}}
\end{array}\right)
$$




$$
\begin{aligned}
\mathbf{B}\left(r(t), r_{1}(t), r_{2}(t), r_{3}(t), d_{1}(t), d_{2}(t)\right) \\
=\left(\begin{array}{cccc}
\frac{-1}{J_{1}+J_{2}} & \frac{1}{J_{1}} & 0 & \frac{-1}{J_{1}} \\
-\frac{1}{J_{1}+J_{2}} & 0 & \frac{1}{J_{2}} & \frac{1}{J_{2}}
\end{array}\right)\left(\begin{array}{l}
r(t) \\
r_{1}(t) \\
r_{2}(t) \\
r_{3}(t)
\end{array}\right) \\
\quad+\left(\begin{array}{cc}
\frac{J_{2}}{J_{1} \cdot\left(J_{1}+J_{2}\right)} & -\frac{1}{J_{1}+J_{2}} \\
-\frac{1}{J_{1}+J_{2}} & \frac{J_{1}}{J_{1} \cdot\left(J_{1}+J_{2}\right)}
\end{array}\right)\left(\begin{array}{l}
d_{1}(t) \\
d_{2}(t)
\end{array}\right) .
\end{aligned}
$$

To achieve asymptotic stability, every eigenvalue of $\mathbf{A}$ should have a strictly negative real part, according to Lyapunov stability theory [25]. In terms of the feedback control gains, the stability conditions for the second-order system (34) are derived as

$$
\begin{aligned}
& k_{11}-k_{31}+k_{21}-k_{32}<0 \\
& k_{21}^{2}+k_{21}\left(k_{31}-k_{32}\right)+k_{22}\left(k_{31}-k_{11}\right)-k_{11} k_{32}<0 .
\end{aligned}
$$

\section{B. Parameter Selection}

For the overactuated system with three inputs $\left(u_{1}(t) u_{2}(t) u_{3}(t)\right)$ and two outputs $\left(y_{p 1}(t) y_{p 2}(t)\right)$, there are theoretically countless solutions of $\left(u_{1}(t) u_{2}(t) u_{3}(t)\right)$ that are able to achieve the desired outputs. If one of the three inputs is predefined, then the input combination of the other two has only one solution. These combinations with one predefined input are denoted as $\left(u_{1} u_{2}\right),\left(u_{1} u_{3}\right)$, and $\left(u_{2} u_{3}\right)$, respectively. They are considered as particular solutions of the general combination $\left(\begin{array}{lll}u_{1} & u_{2} & u_{3}\end{array}\right)$. The controller design explored in this paper includes three ingredients: 1) the selection of a suitable combination of control inputs; 2 ) the proper choice of three feedforward variables $r_{i}(t)(i=1,2,3)$; and 3$)$ the determination of the feedback parameters $k_{i j}(i=1,2,3 j=1,2)$.

1) Input Combination Selection: Since the traction motor is the main power source of the vehicle during the mode transition of interest, $u_{2}(t)$ is not supposed to be zero. Therefore, the input combination $\left(u_{1}(t) u_{3}(t)\right)$ is not used. The other three combinations are $\left(u_{1}(t) u_{2}(t)\right),\left(u_{2}(t) u_{3}(t)\right)$, and $\left(u_{1}(t) u_{2}(t) u_{3}(t)\right)$. They reflect different strategies in varying $u_{1}(t)$ and $u_{3}(t)$.

The engine torque is usually small during the mode transition under investigation. Note that a small engine torque tends to fall into a low-efficiency area of the engine operation, as less usage of $u_{1}(t)$ is helpful for improving engine fuel efficiency. For the clutch usage, smaller friction torque and shorter friction duration come with less frictional losses, suggesting that less usage of $u_{3}(t)$ is beneficial for the driveline efficiency and clutch lifetime.

The combinations $\left(u_{1}(t) u_{2}(t)\right)$ and $\left(u_{2}(t) u_{3}(t)\right)$ are special cases of $\left(u_{1}(t) u_{2}(t) u_{3}(t)\right)$, with $u_{3}(t)=0$ and $u_{1}(t)=0$, respectively. The former does not produce any frictional loss but forces the engine to operate at the points corresponding to low efficiency. The latter avoids low-efficiency operation of the engine but causes higher clutch frictional loss. A good tradeoff between the two extreme cases is necessary to achieve a good balance between high fuel efficiency and low component wearing.

This paper takes the viewpoint that reducing frictional losses is more important than achieving high engine efficiency during mode transitions. One reason is that large friction torque and long engagement durations may result in substantial frictional losses [26], which substantially reduces the clutch life span. The other reason is that the influence of the mode transition on the engine efficiency for a whole driving cycle is often inconsequential, given that most transitions are completed in only a few seconds and the time spent in transition is a relatively small fraction of the entire engine operation. Therefore, it is reasonable to avoid $u_{3}(t)$ and apply the combination of $\left(u_{1}(t) u_{2}(t)\right)$ as much as possible from the beginning of the mode transition and then introduce $u_{3}(t)$ to quickly engage the clutch with a small friction torque when the relative speed of the two clutch shafts is below $\varepsilon$, which is a small threshold value chosen by the designer. The algorithm for the input combination selection is then expressed as

$$
\begin{aligned}
& \text { if abs }\left(\omega_{1}(t)-\omega_{2}(t)\right)>\varepsilon \text { then } \\
& \text { STEP 1: }\left(u_{1}(t) \quad u_{2}(t)\right) \text { is selected } \\
& \text { else } \\
& \text { STEP 2: }\left(u_{1}(t) \quad u_{2}(t) \quad u_{3}(t)\right) \text { is selected }
\end{aligned}
$$$$
\text { end. }
$$

2) Feed-Forward Design: From (39), the three feed-forward variables or the reference inputs $r_{1}(t), r_{2}(t)$, and $r_{3}(t)$ do not

$$
\begin{aligned}
& \left(\begin{array}{c}
\dot{e}_{1}(t) \\
\dot{e}_{2}(t)
\end{array}\right)=\left(\begin{array}{c}
\frac{u_{1}(t)-u_{3}(t)+d_{1}(t)}{J_{1}}-\frac{u_{m}(t)+d_{1}(t)+d_{2}(t)}{J_{1}+J_{2}} \\
\frac{u_{2}(t)+u_{3}(t)+d_{2}(t)}{J_{2}}-\frac{u_{m}(t)+d_{1}(t)+d_{2}(t)}{J_{1}+J_{2}}
\end{array}\right) \\
& =\left(\begin{array}{c}
\frac{r_{1}(t)+k_{11} e_{1}(t)+k_{12} e_{2}(t)-\left(r_{3}(t)+k_{31} e_{1}(t)+k_{32} e_{2}(t)\right)+d_{1}(t)}{J_{1}}-\frac{r(t)+d_{1}(t)+d_{2}(t)}{J_{1}+J_{2}} \\
\frac{r_{2}(t)+k_{21} e_{1}(t)+k_{22} e_{2}(t)+r_{3}(t)+k_{31} e_{1}(t)+k_{32} e_{2}(t)+d_{2}(t)}{J_{2}}-\frac{r(t)+d_{1}(t)+d_{2}(t)}{J_{1}+J_{2}}
\end{array}\right) \\
& =\left(\begin{array}{ll}
\frac{k_{11}-k_{31}}{J_{1}} & \frac{k_{21}-k_{32}}{J_{1}} \\
\frac{k_{21}+k_{31}}{J_{2}} & \frac{k_{22}+k_{32}}{J_{2}}
\end{array}\right)\left(\begin{array}{c}
e_{1}(t) \\
e_{2}(t)
\end{array}\right)+\left(\begin{array}{cccc}
\frac{-1}{J_{1}+J_{2}} & \frac{1}{J_{1}} & 0 & \frac{-1}{J_{1}} \\
-\frac{1}{J_{1}+J_{2}} & 0 & \frac{1}{J_{2}} & \frac{1}{J_{2}}
\end{array}\right)\left(\begin{array}{c}
r(t) \\
r_{1}(t) \\
r_{2}(t) \\
r_{3}(t)
\end{array}\right)+\left(\begin{array}{cc}
\frac{J_{2}}{J_{1} \cdot\left(J_{1}+J_{2}\right)} & -\frac{1}{\left(J_{1}+J_{2}\right)} \\
-\frac{1}{J_{1}+J_{2}} & \frac{J_{1}}{J_{1} \cdot\left(J_{1}+J_{2}\right)}
\end{array}\right)\left(\begin{array}{l}
d_{1}(t) \\
d_{2}(t)
\end{array}\right) \\
& =\mathbf{A}\left(\begin{array}{l}
e_{1}(t) \\
e_{2}(t)
\end{array}\right)+\mathbf{B}\left(r(t), r_{1}(t), r_{2}(t), r_{3}(t), d_{1}(t), d_{2}(t)\right)
\end{aligned}
$$


affect the system stability but will influence the output response $\mathbf{e}(t)$. The main function of the feed-forward regulator is to reduce or avoid disturbance on the zero-input response, so that the exogenous term $\mathbf{B}\left(r(t), r_{1}(t), r_{2}(t), r_{3}(t), d_{1}(t), d_{2}(t)\right)$ in (37) can reinforce the error convergence. The feed-forward variables $r_{1}(t), r_{2}(t)$, and $r_{3}(t)$ are therefore subject to the constraints

$$
\begin{aligned}
& \frac{-r(t)}{J_{1}+J_{2}}+\frac{r_{1}(t)}{J_{1}}-\frac{r_{3}(t)}{J_{1}}+\frac{J_{2} \cdot d_{1}(t)}{J_{1} \cdot\left(J_{1}+J_{2}\right)}-\frac{d_{2}(t)}{J_{1}+J_{2}}=0 \\
& \frac{-r(t)}{J_{1}+J_{2}}+\frac{r_{2}(t)}{J_{2}}+\frac{r_{3}(t)}{J_{2}}-\frac{d_{1}(t)}{J_{1}+J_{2}}+\frac{J_{1} \cdot d_{2}(t)}{J_{2} \cdot\left(J_{1}+J_{2}\right)}=0 .
\end{aligned}
$$

Eliminating $r_{3}(t)$ and combining the two equations, a constraint equation for $r_{1}(t)$ and $r_{2}(t)$ can be derived as

$$
r_{1}(t)+r_{2}(t)=r(t)
$$

One notices that (45) involves only two variables. We now have one DoF in the design of the feed-forward control. While infinitely many solutions exist to meet the constraint equations, we will leverage the extra DoF to adapt and optimize the performance for various vehicle operating scenarios and different design specifications.

The calculation of two extreme cases $r_{1}(t)=0$ and $r_{3}(t)=$ 0 are given here as examples.

When $r_{1}(t)=0, r_{2}(t)$ and $r_{3}(t)$ are calculated as

$$
\begin{aligned}
& r_{2}(t)=r(t) \\
& r_{3}(t)=-\frac{J_{1}}{J_{1}+J_{2}} r(t)+\frac{J_{2}}{J_{1}+J_{2}} d_{1}(t)-\frac{J_{1}}{J_{1}+J_{2}} d_{2}(t) .
\end{aligned}
$$

When $r_{3}(t)=0, r_{1}(t)$ and $r_{2}(t)$ are calculated as

$$
\begin{aligned}
& r_{1}(t)=\frac{J_{1}}{J_{1}+J_{2}} r(t)-\frac{J_{2}}{J_{1}+J_{2}} d_{1}(t)+\frac{J_{1}}{J_{1}+J_{2}} d_{2}(t) \\
& r_{2}(t)=\frac{J_{2}}{J_{1}+J_{2}} r(t)+\frac{J_{2}}{J_{1}+J_{2}} d_{1}(t)-\frac{J_{1}}{J_{1}+J_{2}} d_{2}(t) .
\end{aligned}
$$

3) Selection of Feedback Parameters: With six feedback parameters and two stability conditions (40) and (41), we have four extra DoFs left that we can explore for better performance and a simpler algorithm. From (27)-(29) and (31), $e_{2}(t)$ is found to be independent of $u_{1}(t)$; it is therefore reasonable to regulate $u_{1}(t)$ using only the feedback from $e_{1}(t)$. Similarly, $e_{1}(t)$ is independent of $u_{2}(t)$, so $u_{2}(t)$ can use only the feedback from $e_{2}(t)$. Hence, parameters $k_{12}$ and $k_{21}$ are set to be zero, reducing two DoFs. The stability conditions (40) and (41) are then simplified as

$$
\begin{aligned}
& C_{1}=k_{11}-\left(k_{31}+k_{32}\right)<0 \\
& C_{2}=k_{22}\left(k_{31}-k_{11}\right)-k_{11} k_{32}<0 .
\end{aligned}
$$

There are still two DoFs in the selection of the four parameters. One technique for $k_{22}$ selection is given here to illustrate what can be achieved by exploring this design space. Based on the understanding of the underlying physical system operation, $u_{3}(t)$ changes from zero to nonzero during the HEV mode transition and can be considered as a disturbance to state variable $x_{p 2}(t)$. Therefore, the state equation (28) can be rewritten as

$$
J_{2} \dot{x}_{p 2}(t)=r_{2}(t)+k_{21} e_{1}(t)+k_{22} e_{2}(t)+u_{3}(t)+d_{2}(t) .
$$

Input $u_{2}(t)$ has two parts: one is the reference input $r_{2}(t)$, and the other is the feedback regulator $k_{21} e_{1}(t)+k_{22} e_{2}(t)$. If $u_{3}(t)$ counteracts the second part of $u_{2}(t)$, then $x_{p 2}(t)$ follows the trajectory determined by $r_{2}(t)$, and the disturbance introduced by $u_{3}(t)$ is mitigated. With $k_{21}$ being set to zero, a reference value of $k_{22}$ is proposed as

$$
k_{22 \_r}(t)=-\frac{u_{3}(t)}{e_{2}(t)} .
$$

In addition, the calculation of $k_{22}$ is an offset of $k_{22} e$ relative to $k_{22} r$

$$
k_{22}(t)=k_{22 \_r}(t)+k_{22 \_e} .
$$

Thus, $k_{22}(t)$ introduces a control variable to the system, making it a time-varying system and therefore making the stability criterion (40) and (41) no longer applicable. However, the selection of $k_{22}$ e makes $k_{22}(t)$ satisfy the criterion designed for the nonlinear time-varying system [27]. The detailed derivation is not included in the scope of this paper.

\section{Continuity Analysis for $u_{3}(t)$}

Generally speaking, $u_{1}(t)$ and $u_{2}(t)$ can be continuously actuated throughout the mode transition process of the SPHEV, whereas $u_{3}(t)$ may be discontinuous due to slip-stick dynamics when the clutch makes the transition from the slipping phase to the locked phase. Using the control law proposed in this paper, a continuous $u_{3}(t)$ is worked out for slip-stick transition. The continuity is proved in this section.

If $u_{3}(t)$ at the end of the slipping phase approaches the value of the upcoming stick phase, then a continuous slip-stick transition is achieved. At the end of the slipping phase, $u_{3}(t)$ is denoted by $u_{3}^{-}(t)$ and is calculated from (34) as

$$
u_{3}^{-}(t)=r_{3}(t)+k_{31} e_{1}(t)+k_{32} e_{2}(t) \text {. }
$$

The clutch friction torque $T_{c}$ in the locked phase is given in (15). Let $u_{1}^{+}(t), u_{2}^{+}(t)$, and $u_{3}^{+}(t)$ denote the control inputs in the locked phase, respectively. Substitution of (20) into (15) yields

$$
\begin{aligned}
u_{3}^{+}(t)=\frac{J_{2}}{J_{1}+J_{2}} u_{1}^{+}(t) & -\frac{J_{1}}{J_{1}+J_{2}} u_{2}^{+}(t) \\
& +\frac{J_{2}}{J_{1}+J_{2}} d_{1}(t)-\frac{J_{1}}{J_{1}+J_{2}} d_{2}(t) .
\end{aligned}
$$


Applying the MRC control law in (32) and (33) to (56), we have

$$
\begin{aligned}
u_{3}^{+}(t)= & \frac{J_{2}}{J_{1}+J_{2}}\left(r_{1}(t)+k_{11} e_{1}(t)\right) \\
& -\frac{J_{1}}{J_{1}+J_{2}}\left(r_{2}(t)+k_{22} e_{2}(t)\right) \\
& +\frac{J_{2}}{J_{1}+J_{2}} d_{1}(t)-\frac{J_{1}}{J_{1}+J_{2}} d_{2}(t) \\
= & \frac{J_{2}}{J_{1}+J_{2}} r_{1}(t)-\frac{J_{1}}{J_{1}+J_{2}} r_{2}(t)+\frac{J_{2}}{J_{1}+J_{2}} d_{1}(t) \\
& -\frac{J_{1}}{J_{1}+J_{2}} d_{2}(t)+\frac{J_{2}}{J_{1}+J_{2}} k_{11} e_{1}(t) \\
& -\frac{J_{1}}{J_{1}+J_{2}} k_{22} e_{2}(t) .
\end{aligned}
$$

Continuity of $u_{3}(t)$ implies $u_{3}^{-}(t)=u_{3}^{+}(t)$; therefore, if the following equation is satisfied

$$
\begin{aligned}
r_{3}(t) & +k_{31} e_{1}(t)+k_{32} e_{2}(t) \\
= & \frac{J_{2}}{J_{1}+J_{2}} r_{1}(t)-\frac{J_{1}}{J_{1}+J_{2}} r_{2}(t)+\frac{J_{2}}{J_{1}+J_{2}} d_{1}(t) \\
& -\frac{J_{1}}{J_{1}+J_{2}} d_{2}(t)+\frac{J_{2}}{J_{1}+J_{2}} k_{11} e_{1}(t)-\frac{J_{1}}{J_{1}+J_{2}} k_{22} e_{2}(t)
\end{aligned}
$$

then continuity is assured. Exploring the design freedom in the control, we divide (58) into two parts and force the equations

$$
\begin{aligned}
r_{3}(t)= & \frac{J_{2}}{J_{1}+J_{2}} r_{1}(t)-\frac{J_{1}}{J_{1}+J_{2}} r_{2}(t) \\
& +\frac{J_{2}}{J_{1}+J_{2}} d_{1}(t)-\frac{J_{1}}{J_{1}+J_{2}} d_{2}(t)(59) \\
k_{31} e_{1}(t)+k_{32} e_{2}(t)= & \frac{J_{2}}{J_{1}+J_{2}} k_{11} e_{1}(t)-\frac{J_{1}}{J_{1}+J_{2}} k_{22} e_{2}(t) .
\end{aligned}
$$

For the first part, the equality in (59) is guaranteed if $r_{1}(t)$ and $r_{2}(t)$ are selected according to the constraints in (43) and (44), i.e., we choose $r_{1}(t)$ and $r_{2}(t)$ as

$$
\begin{aligned}
& r_{1}(t)=r_{3}(t)-\frac{J_{2} \cdot d_{1}(t)}{J_{1}+J_{2}}+\frac{J_{1} \cdot d_{2}(t)}{J_{1}+J_{2}}+\frac{J_{1} \cdot r(t)}{J_{1}+J_{2}} \\
& r_{2}(t)=-r_{3}(t)+\frac{J_{2} \cdot d_{1}(t)}{J_{1}+J_{2}}-\frac{J_{1} \cdot d_{2}(t)}{\left(J_{1}+J_{2}\right)}+\frac{J_{2} \cdot r(t)}{J_{1}+J_{2}} .
\end{aligned}
$$

Substitution of (61) and (62) yields

$$
\begin{aligned}
& \frac{J_{2}}{J_{1}+J_{2}} r_{1}(t)-\frac{J_{1}}{J_{1}+J_{2}} r_{2}(t)+\frac{J_{2}}{J_{1}+J_{2}} d_{1}(t)-\frac{J_{1}}{J_{1}+J_{2}} d_{2}(t) \\
&=\frac{J_{2}}{J_{1}+J_{2}}\left(r_{3}(t)-\frac{J_{2} \cdot d_{1}(t)}{J_{1}+J_{2}}+\frac{J_{1} \cdot d_{2}(t)}{J_{1}+J_{2}}+\frac{J_{1} \cdot r(t)}{J_{1}+J_{2}}\right) \\
& \quad-\frac{J_{1}}{J_{1}+J_{2}}\left(-r_{3}(t)+\frac{J_{2} \cdot d_{1}(t)}{J_{1}+J_{2}}-\frac{J_{1} \cdot d_{2}(t)}{\left(J_{1}+J_{2}\right)}+\frac{J_{2} \cdot r(t)}{J_{1}+J_{2}}\right) \\
& \quad+\frac{J_{2}}{J_{1}+J_{2}} d_{1}(t)-\frac{J_{1}}{J_{1}+J_{2}} d_{2}(t) \\
&= r_{3}(t)
\end{aligned}
$$

which proves (59).
Equation (60) can be rewritten as

$$
\left(\begin{array}{ll}
k_{31} & k_{32}
\end{array}\right) \cdot \mathbf{e}(t)=\left(\frac{J_{2} k_{11}}{J_{1}+J_{2}}-\frac{J_{1} k_{22}}{J_{1}+J_{2}}\right) \cdot \mathbf{e}(t) .
$$

If $\mathbf{e}(t)=0$, any $k_{31}$ and $k_{32}$ that satisfies the stability conditions are solutions of the aforementioned equation. $\mathbf{e}(t)=0$ implies that the clutch is synchronized and running at the same speed with the reference model when the clutch just finishes the slipping phase and begins the locked phase.

On the other hand, if $\mathbf{e}(t) \neq 0$, no suitable solution satisfies both the continuity and stable conditions. The only solution that meets the continuity condition is

$$
\left(\begin{array}{ll}
k_{31} & k_{32}
\end{array}\right)=\left(\frac{J_{2} k_{11}}{J_{1}+J_{2}}-\frac{J_{1} k_{22}}{J_{1}+J_{2}}\right) .
$$

Substitution of (65) into (50) and (51) yields

$$
\begin{aligned}
k_{11}+k_{22} & <0 \\
k_{22}\left(\frac{J_{2} k_{11}}{J_{1}+J_{2}}-k_{11}\right)+k_{11} \frac{J_{1} k_{22}}{J_{1}+J_{2}} & =0 .
\end{aligned}
$$

The equality in (67) shows that the system is critical stable but is not strictly asymptotical stable, as required by the inequality condition (51). Therefore, this is not a suitable solution.

In summary, the continuity of $u_{3}(t)$ is achieved under two conditions: with the feed-forward variables $r_{1}(t)$ and $r_{2}(t)$ satisfying constraints (43) and (44), and with $\mathbf{e}(t)=0$ at the end of the slipping phase.

\section{RESUlts AND Discussions}

Many hybrid bus drivers have observed that, by pressing the accelerator pedal gradually and then quickly releasing the clutch pedal during the mode transition from motor-only to compound driving, the transition time and power interruption is reduced [15]. This heuristic operating strategy will be referred to as the conventional operation in this paper, and the results are used as the baseline for assessing the proposed MRC performance. This section includes three parts: 1) a simulation study to compare the results of the proposed MRC and the conventional operation; 2) sensitivity investigation of the selection of variables and parameters on the mode transition performance; and 3) experimental validation of the MRC used in an SPHEV bus.

Simulations are performed first on an SPHEV bus model with parameters listed in Table I. In addition, the estimated value of $T_{f}(t)$ is $20 \mathrm{~N} \cdot \mathrm{m}$. To reflect real-world physical constraints, the change rates of the engine torque and clutch torque are limited between $-100-30 \mathrm{~N} \cdot \mathrm{m} / \mathrm{s}$ and $-200-100 \mathrm{~N} \cdot \mathrm{m} / \mathrm{s}$, respectively, in simulations. Meanwhile, no additional rate limitation is imposed on the motor torque because of its fast response.

\section{A. Comparison With Conventional Method}

To compare the performance of the two mode transition strategies, we consider the scenario of a typical startup process 
TABLE I

PARAMETERS FOR SIMULATION

\begin{tabular}{llll}
\hline \hline Parameter & Value & Parameter & \multicolumn{1}{c}{ Value } \\
\hline$J_{\text {eng }}$ & $1.0 \mathrm{~kg} \cdot \mathrm{m}^{2}$ & $R$ & $0.525 \mathrm{~m}$ \\
$J_{\text {isg }}$ & $0.9 \mathrm{~kg} \cdot \mathrm{m}^{2}$ & $f$ & 0.0015 \\
$m$ & $16000 \mathrm{~kg}$ & $c_{D}$ & 0.32 \\
$J_{T M}$ & $0.88 \mathrm{~kg} \cdot \mathrm{m}^{2}$ & $A_{V}$ & $1.8 \mathrm{~m}^{2}$ \\
$J_{\text {cltch }}$ & $0.05 \mathrm{~kg} \cdot \mathrm{m}^{2}$ & $\rho_{\text {air }}$ & $1.205 \mathrm{~kg} / \mathrm{m}^{3}$ \\
$J_{\text {fst_gear }}$ & $0.05 \mathrm{~kg} \cdot \mathrm{m}^{2}$ & $N$ & 2 \\
$J_{\text {fnl_gear }}$ & $0.05 \mathrm{~kg} \cdot \mathrm{m}^{2}$ & $\mu_{s}$ & 0.40 \\
$T_{\text {tm_max }}$ & $450 \mathrm{Nm}$ & $\mu_{l}$ & 0.44 \\
$i_{0}$ & 5.571 & $R_{c}$ & $0.15 \mathrm{~m}$ \\
$i_{1}$ & 3.78 & $A$ & $0.038 \mathrm{~m}^{2}$ \\
$i_{\text {tm }}$ & 4 & $\alpha$ & 0 \\
\hline \hline
\end{tabular}

of the SPHEV bus. The desired vehicle acceleration is positive. The results of the proposed MRC and the conventional operation (abbreviated by Conv.) are shown in Fig. 5.

The mode transition begins when the angular speed of Shaft B reaches $140.0 \mathrm{rad} / \mathrm{s}$, i.e., the vehicle velocity reaches $12.6 \mathrm{~km} / \mathrm{h}$. The initial angular speed of the engine is supposed to be $50 \mathrm{rad} / \mathrm{s}$, which is reached by a starter. The values of the control variables and parameters used in algorithm (42) for the two steps are listed in Table II. Since $r_{1}(t)$ is predefined, $r_{2}(t)$ and $r_{3}(t)$ are calculated to satisfy (45) and (43), respectively.

The algorithm of the conventional operation is given by

$$
u_{1}(t)=g_{1} \cdot t, \quad u_{2}(t)=\bar{T}_{\text {tm_cmd }}, \quad u_{3}(t)=g_{3} \cdot t
$$

in which $g_{1}=30$ and $g_{3}=500$ are chosen for the simulation.

The mode transition duration of the MRC is $3 \mathrm{~s}$ longer than that of the Conv., as shown in Fig. 5(e). However, as shown in Fig. 5(d), the slipping friction duration of the MRC is $1.30 \mathrm{~s}$ (from 13.27 to $14.55 \mathrm{~s}$ ), whereas that of the Conv. is $1.368 \mathrm{~s}$ (from 9.36 to $10.79 \mathrm{~s}$ ), so the former is $0.068 \mathrm{~s}$ shorter. Note that the clutch friction torque of the Conv. is actuated earlier and with much higher intensity, so it quickly engages the two clutch shafts. This is the main reason that, in comparison, the MRC has a longer transition. Nevertheless, fast transition does not mean short clutch engagement for the SPHEV mode transition. The MRC reduces slipping time and friction torque, thereby enhancing performance, as discussed in the succeeding paragraphs.

Driveline torque interruption, vehicle jerk, and frictional losses are evaluated for performance assessment. Driveline torque interruption is quantitatively captured by the vehicle acceleration. Vehicle jerk is the index for smoothness, which is measured by the derivative of the vehicle acceleration [22]. Frictional losses are calculated using the following formula [22]:

$$
E_{D}=\int_{t_{1}}^{t_{2}} \operatorname{abs}\left(\omega_{1}-\omega_{2}\right) \cdot T_{c} d t .
$$

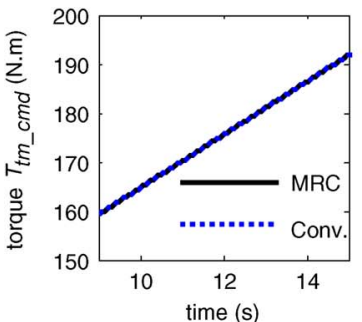

(a)

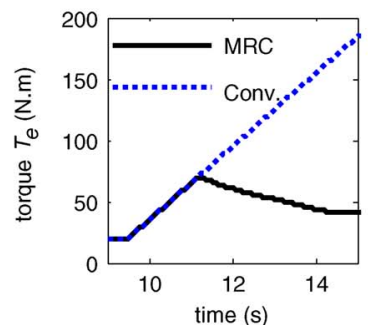

(c)

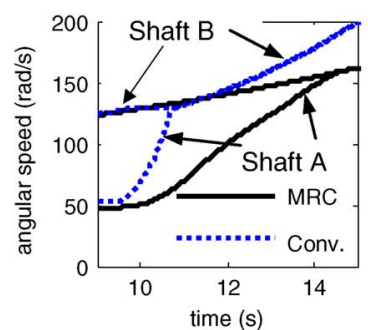

(e)

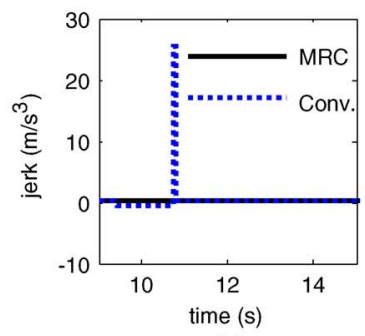

(g)

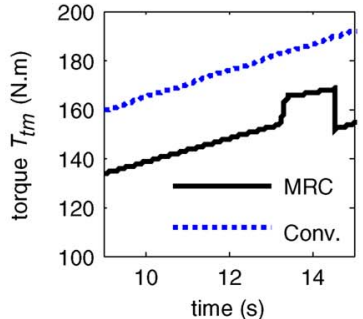

(b)

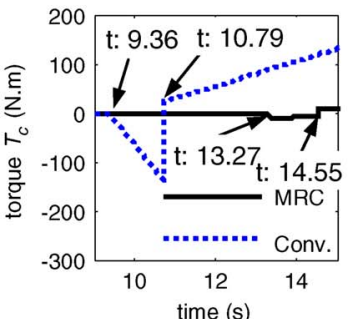

(d)

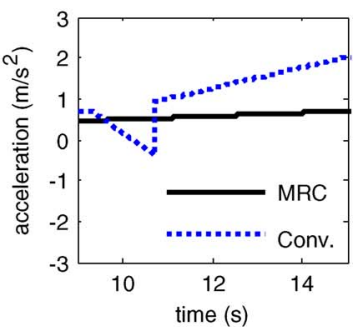

(f)

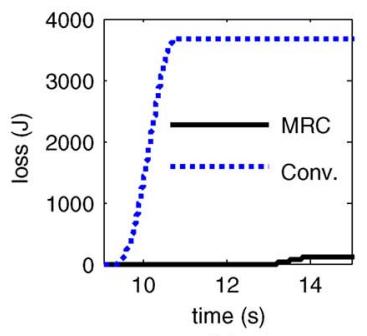

(h)
Fig. 5. Comparison between MRC and conventional operation: simulations. (a) Desired traction torque $T_{\mathrm{tm} \text { cmd }}$. (b) Traction motor torque $T_{\mathrm{tm}}$. (c) Engine torque $T_{e}$. (d) Clutch torque $T_{c}$. (e) Angular speed $\omega_{1}$ and $\omega_{2}$. (f) Vehicle acceleration. (g) Vehicle jerk. (h) Frictional losses $E_{D}$.

The vehicle acceleration of the MRC is maintained, whereas that of the Conv. falls by $1.3 \mathrm{~m} / \mathrm{s}^{2}$ at the end of the slipping phase, as shown in Fig. 5(f). For the Conv., the profile of the vehicle acceleration is similar to that of the clutch friction torque, which implies that the clutch torque negatively affects the vehicle acceleration. Moreover, the motor torque does not compensate this negative effect, as shown in Fig. 5(c). Thus, the vehicle acceleration has to fall. However, the MRC avoids the negative effect in two ways: First, the clutch friction torque is much smaller, which translates to a smaller negative effect on the vehicle acceleration; second, the MRC increases the traction motor torque to compensate the negative effect.

No substantial vehicle jerk occurs, even in the slip-stick transition, in the MRC-based control simulation. However, a sudden vehicle jerk of over $20 \mathrm{~m} / \mathrm{s}^{3}$ is found in the results from the Conv., as shown in Fig. 5(g). The reason for the sudden jerk is that the clutch friction torque sharply changes because of a slip-stick friction transition at this point. The Conv. quickly 
TABLE II

Control VARIABles AND PARAMETERs

\begin{tabular}{lll}
\hline \hline Parameter or variable & \multicolumn{1}{r}{ STEP 1 } & STEP 2 \\
\hline$r_{1}(t)$ & 40 & 40 \\
$r_{2}(t)$ & calculated & calculated \\
$r_{3}(t)$ & 0 & calculated \\
$k_{11}$ & -0.5 & -0.5 \\
$k_{12}$ & 0 & 0 \\
$k_{21}$ & 0 & 0 \\
$k_{22} e$ & -30 & -30 \\
$k_{31}$ & 0 & -0.1 \\
$k_{32}$ & 0 & -0.1 \\
$\varepsilon$ & $20 \mathrm{rad} / \mathrm{s}$ \\
\hline \hline
\end{tabular}

increases the clutch torque. The torque becomes large at the end of the slipping phase and is much larger than the torque in the upcoming locked phase. Thereafter, the friction torque suddenly changes, and an intensive jerk occurs. For the MRC, the clutch friction torque in the slipping phase is controlled to approach the reference model, which has a locked clutch; moreover, the torque continuity is analyzed and guaranteed in Section IV-C. Thus, the MRC avoids a sudden change of the friction torque and the resulting jerk.

The frictional losses resulting from the MRC operation are only about $112 \mathrm{~J}$, whereas up to $3651 \mathrm{~J}$ are lost during conventional operation. The frictional losses are produced in the slipping phase and are affected by three factors according to (69): the angular speed difference of the two clutch shafts, the friction torque, and the slipping duration. From Fig. 5(d) and (e), all three factors are much less pronounced with MRC than with the Conv. Therefore, the frictional loss is greatly reduced.

In summary, the MRC simulation shows no torque interruption, no sudden jerk, and little frictional loss. These results suggest that an MRC-based system is beneficial for vehicle running performance and also for energy efficiency and clutch lifetime span.

\section{B. Sensitivity Analysis}

To further understand the design implications of the MRC strategy, we considered the key factors that influence the mode transition performance including the following: the three feedforward variables $r_{1}(t), r_{2}(t)$, and $r_{3}(t)$; the switch trigger $\varepsilon$ in algorithm (42); the estimation errors of the external disturbances $\bar{T}_{r}(t)$ and $T_{f}(t)$; and the actuation noise of the engine torque $T_{e}(t)$ and clutch torque $T_{c}(t)$. The analysis of effects due to various factors is performed through simulations.

1) Feed-Forward Selection $\left(r_{1}(t), r_{2}(t), r_{3}(t)\right)$ : The selection principles for $r_{1}(t), r_{2}(t)$, and $r_{3}(t)$ allow for one DoF for the three feed-forward inputs. If any one of the three parameters is defined, then the other two are determined uniquely based on the strategy presented in Section IV-B2. Which variable should be predefined and how to determine its value are relevant questions for real applications. The physical meanings of $r_{1}(t)$ and $r_{2}(t)$ are the engine torque and motor torque when $e_{1}(t)$ and $e_{2}(t)$ are zero. Therefore, $r_{1}(t)$ or $r_{2}(t)$ can be defined

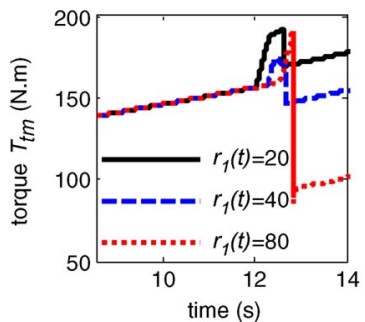

(a)

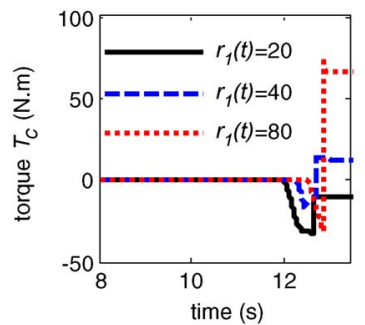

(c)

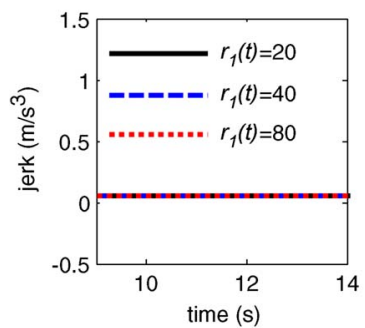

(e)

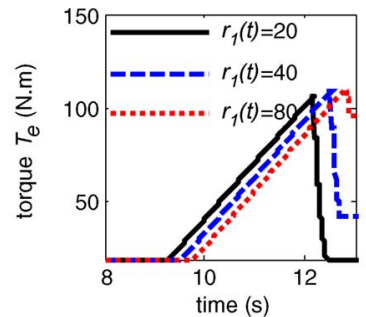

(b)

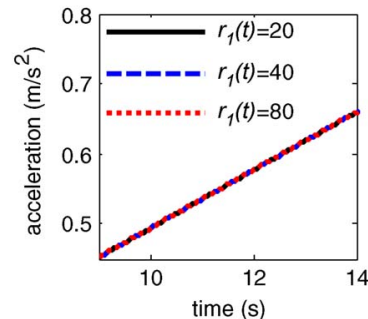

(d)

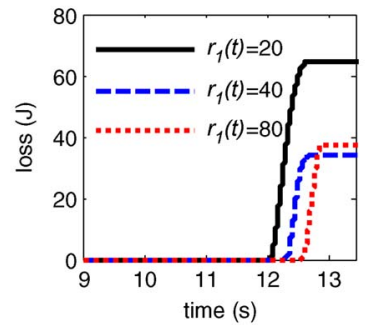

(f)
Fig. 6. Effects of $r_{1}(t)$. (a) Traction motor torque $T_{\mathrm{tm}}$. (b) Engine torque $T_{e}$ (c) Clutch torque $T_{c}$. (d) Vehicle acceleration. (e) Vehicle jerk. (f) Frictional losses $E_{D}$.

according to a torque distribution based on the energy management strategy.

One example for predefined $r_{1}(t)$ is given in Fig. 6. Here, $r_{1}(t)$ is set to be 20,40 , and 80 , whereas the other parameters are the same as those defined in Table II. The desired traction torque is the same as in Fig. 5(a). Fig. 6(b) shows that $T_{e}$ approaches $r_{1}(t)$ after the clutch has been engaged. No acceleration loss is found in Fig. 6(d), and no sudden jerk in Fig. 6(f). Fig. 6(f) shows $r_{1}(t)=20$ produces $30 \mathrm{~J}$ more friction losses than $r_{1}(t)=80$. Nevertheless, the friction losses are insignificant. Hence, the MRC mode transition control yields good performance for all three different values of $r_{1}(t)$.

The results for different predefined values of $r_{2}(t)$ are similar. Therefore, we conclude that the performance of the proposed MRC is not sensitive to different torque distribution strategies, which is a significant feature for SPHEV energy management.

2) Switch Trigger $\varepsilon$ : Three different values of the switch trigger $\varepsilon(20,50$, and $80 \mathrm{rad} / \mathrm{s})$ are used in simulation to assess the sensitivity of the performance to this design parameter. A larger value of $\varepsilon$ leads to the use of the clutch torque at an earlier stage in Fig. 7(a) and (c) to compensate for the motor torque. The MRC prevents acceleration loss and sudden jerk for the three different $\varepsilon$ as shown in Fig. 7(d) and (e). However, the frictional losses are greatly affected by $\varepsilon . \varepsilon=20$ produces only $33 \mathrm{~J}$, whereas $\varepsilon=80$ produces $3135 \mathrm{~J}$. Small $\varepsilon$ implies less usage of clutch torque, so choosing small $\varepsilon$ is helpful for 


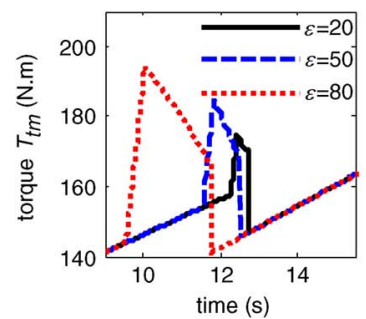

(a)

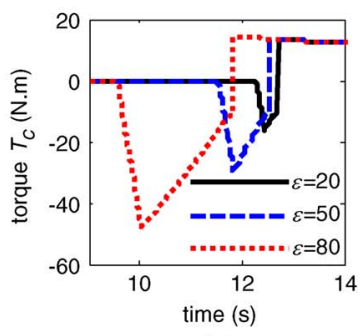

(c)

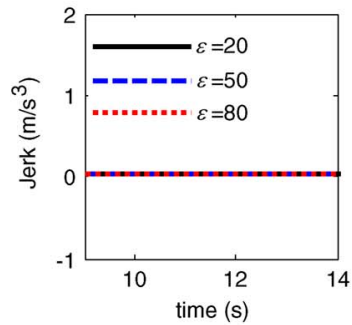

(e) (b)

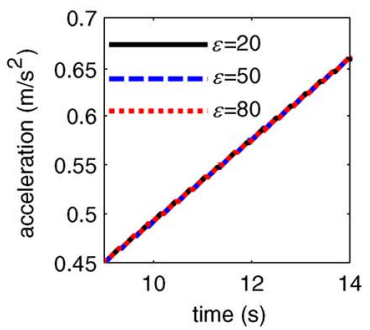

(d)

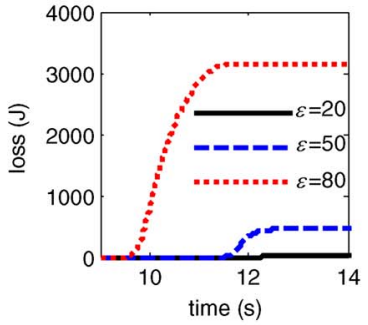

(f)

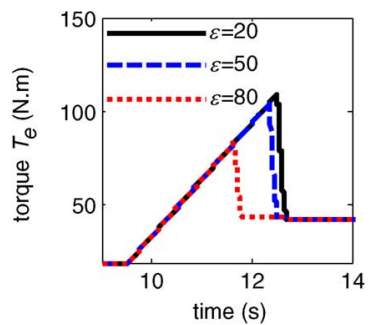

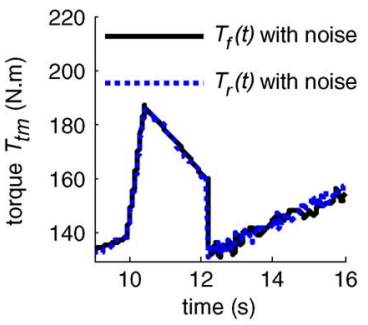

(a)

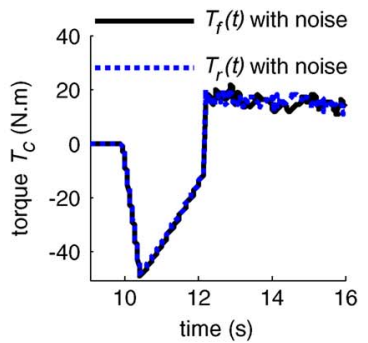

(c)

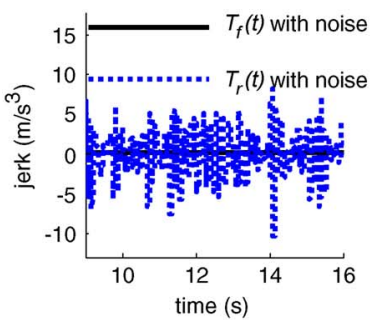

(e)

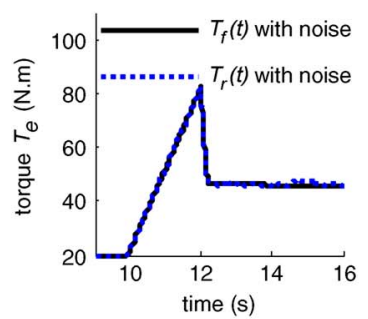

(b)

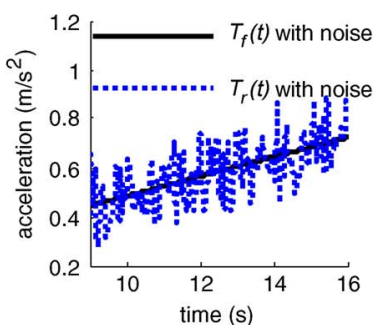

(d)

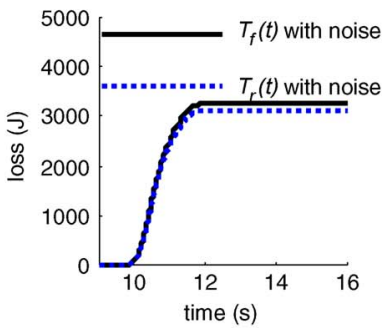

(f)
Fig. 7. Effects of $\varepsilon$. (a) Traction motor torque $T_{\mathrm{tm}}$. (b) Engine torque $T_{e}$ (c) Clutch torque $T_{c}$. (d) Vehicle acceleration. (e) Vehicle jerk. (f) Frictional losses $E_{D}$.

reducing frictional losses. Moreover, small $\varepsilon$ allows for shorter times for the clutch slipping control. The authors found that, in real experiments, it is hard to achieve short time transition with a small $\varepsilon$, particularly in those vehicles with sensor inaccuracy and actuator delay. Consequently, small $\varepsilon$ in those cases causes intensive vehicle jerk at the slip-stick transition. Thus, large $\varepsilon$ is usually used in real applications to avoid this problem. For this reason, most simulations presented in this paper use a large $\varepsilon$.

3) Effects of External Disturbance $T_{f}(t)$ and $T_{r}(t)$ : In real applications, it is hard to accurately measure the external disturbance torques $T_{f}(t)$ and $T_{r}(t)$. Here, the disturbance on $T_{r}(t)$ is calculated using its equivalent torque $\bar{T}_{r}(t)$. Gaussian white noises are applied to $T_{f}(t)$ and $\bar{T}_{r}(t)$ based on the estimated value. The noise bandwidth is $30 \mathrm{~Hz}$ for $T_{f}(t)$ and $20 \mathrm{~Hz}$ for $\bar{T}_{r}(t)$; the noise variance is 6 for $T_{f}(t)$ and 100 for $\bar{T}_{r}(t)$. Thus, the ratio of the noise power to the signal power is $1.63 \%$ for $T_{f}(t)$ and $1.25 \%$ for $T_{r}(t)$, respectively. To show their individual impacts, the two noises are separately applied to the simulations. One simulation uses disturbed $T_{f}(t)$ and ideal $T_{r}(t)$. The other uses ideal $T_{f}(t)$ and disturbed $T_{r}(t)$. The results are given in Fig. 8.

Fig. 8(d)-(f) shows that disturbed $T_{f}(t)$ does not introduce acceleration loss or intensive jerk to the vehicle, whereas disturbed $T_{r}(t)$ introduces apparent vibrations. The reason is that $T_{r}(t)$ directly acts on the vehicle, whereas $T_{f}(t)$ is transmitted to the vehicle through a clutch. Using the designed algorithm,
Fig. 8. Effects of disturbance noise. (a) Traction motor torque $T_{\mathrm{tm}}$. (b) Engine torque $T_{e}$. (c) Clutch torque $T_{c}$. (d) Vehicle acceleration. (e) Vehicle jerk. (f) Frictional losses $E_{D}$.

$T_{f}(t)$ noise is not passed to the vehicle through $T_{c}(t)$, whereas noise on $T_{r}(t)$ has a more straightforward effect on the driveline dynamics.

4) Effects of Actuation Noise of $T_{e}(t)$ and $T_{c}(t)$ : Engine torque and clutch torque are subject to uncertainties due to the physical limitations of their actuation mechanisms. In this study, Gaussian white noises with bandwidths of 30 and $20 \mathrm{~Hz}$ and variances of 80 and 50 are superimposed on clutch torque $T_{c}(t)$ and engine torque $T_{e}(t)$, respectively, resulting in ratios of noise power to signal power of $41.63 \%$ for $T_{c}(t)$ and $22.74 \%$ for $T_{e}(t)$. These high ratios reflect what we have experienced in real applications, particularly for small torque operations. Simulation results of the MRC response with the disturbed torques are shown in Fig. 9.

Due to the clutch actuation disturbance, the dynamical performance is deteriorated in the clutch-slipping stage. Vibration occurs in vehicle acceleration, and the maximum vehicle jerk is up to $6 \mathrm{~m} / \mathrm{s}^{3}$. Thus, the mitigation of the disturbance from clutch actuation is critical to the smooth operation of drivelines. Nevertheless, the impact occurs only in the slipping stage for a short duration. After that, the vehicle acceleration becomes smooth, and the vehicle jerk is small.

The engine torque disturbance has no negative impact in the clutch-slipping stage because the engine torque is separated by the clutch. However, when the clutch is engaged, the engine torque is transmitted down to the vehicle. Then, the motor torque $T_{\mathrm{tm}}$ compensates the disturbance. It is shown in 


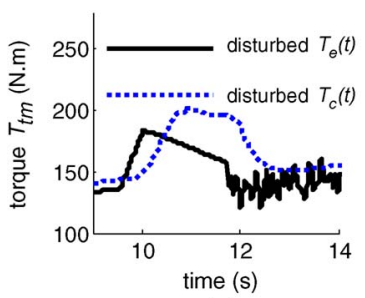

(a)

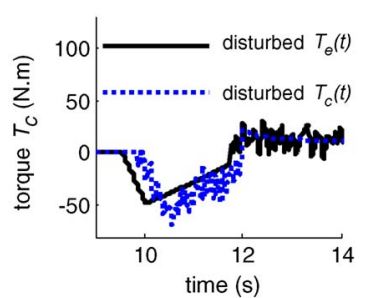

(c)

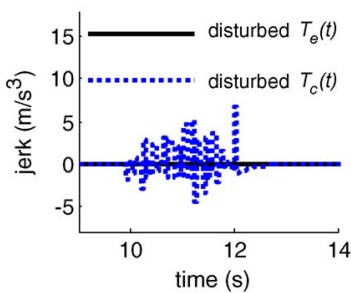

(e)

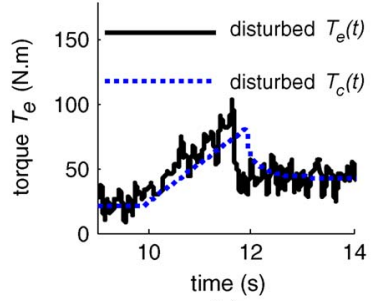

(b)

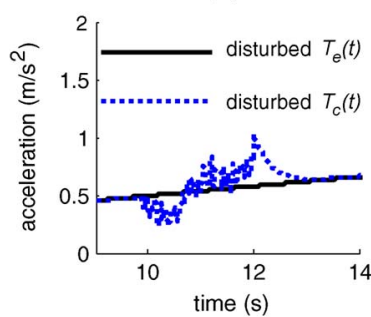

(d)

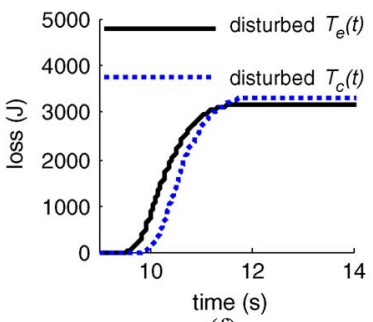

(f)
Fig. 9. Effects of actuation disturbance. (a) Traction motor torque $T_{\mathrm{tm}}$. (b) Engine torque $T_{e}$. (c) Clutch torque $T_{c}$. (d) Vehicle acceleration. (e) Vehicle jerk. (f) Frictional losses $E_{D}$.

Fig. 9(d)-(f) that the MRC compensates the disturbance from engine torque very well.

\section{Experimental Results}

Both the proposed MRC and the Conv. are applied to an SPHEV bus, and the corresponding experimental results are shown in Fig. 10. Even though the results do not exactly match the simulation results, they show the improvement potential of MRC. The mismatch between the experimental and simulation can be attributed to two reasons: one due to the inaccuracy of the model parameters and the other related to discretization and sampling of the digital controller for the clutch actuator. The vehicle acceleration fluctuation, vehicle jerk, and fractional losses are about $0.5 \mathrm{~m} / \mathrm{s}^{2}, 5 \mathrm{~m} / \mathrm{s}^{3}$, and $1573 \mathrm{~J}$, respectively, for the MRC results, compared to $4 \mathrm{~m} / \mathrm{s}^{2}, 25 \mathrm{~m} / \mathrm{s}^{3}$, and $5000 \mathrm{~J}$ for the Conv. Therefore, the mode transition performance for the MRC is much better than that of the Conv. It is observed that jerk appears in the clutch-slipping phase and locked phase, and it is more intensive for the mode transition with conventional methods than with MRC. Note that the Conv. rapidly increases the clutch friction torque in the slipping phase. The fast-increasing torque acts as a large impulse input to the Shaft B driveline with elastic components. Thus, vibration is excited. Moreover, the friction torque suddenly changes due to the slip-stick transition at the end of the slipping phase, i.e., at the beginning of the locked phase. This change produces another large impulse input to the vehicle, and the vibration continues into

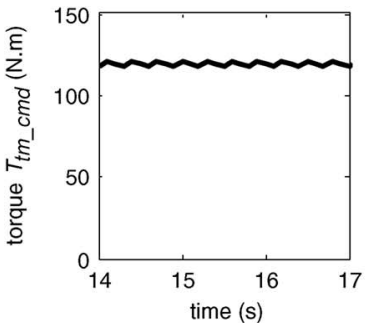

(a)

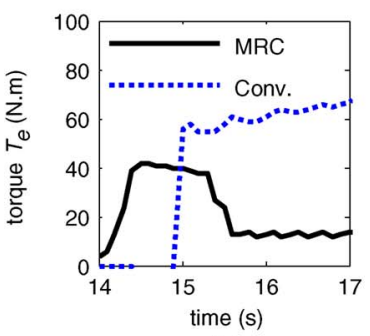

(c)

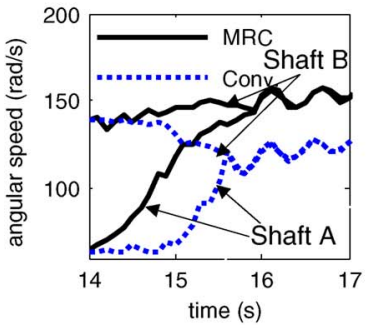

(e)

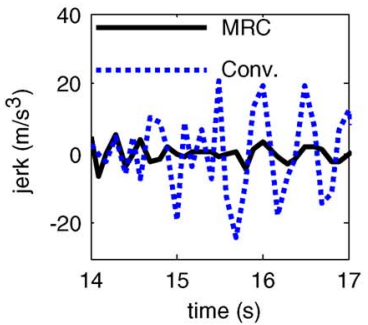

(g)

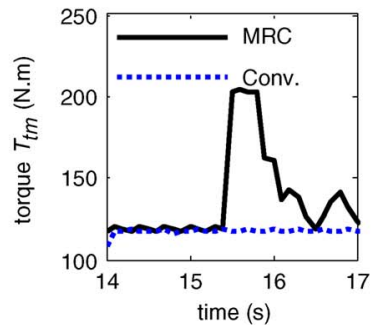

(b)

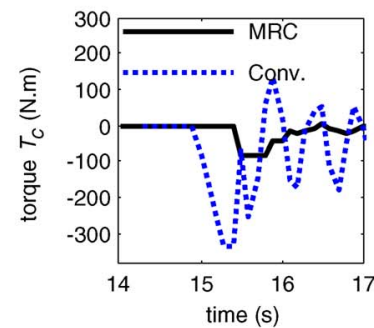

(d)

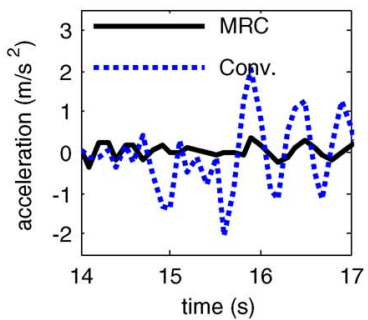

(f)

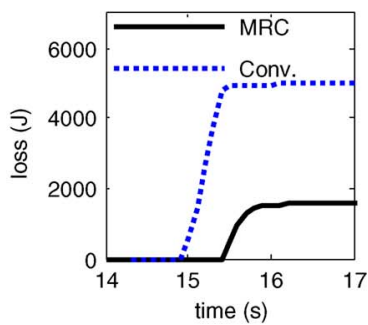

(h)
Fig. 10. Experimental results. (a) Desired traction torque $T_{\text {tm cmd }}$. (b) Traction motor torque $T_{\mathrm{tm}}$. (c) Engine torque $T_{e}$. (d) Clutch torque $T_{c}$. (e) Angular speed $\omega_{1}, \omega_{2}$. (f) vehicle acceleration. (g) Vehicle jerk. (h) Frictional losses $E_{D}$.

the locked phase. For the MRC, although no sudden change in the slip-stick transition is expected in the ideal case, errors due to discretization and sampling in digital controller, as well as limitations in clutch actuation, lead to a relative small impulse input to the driveline, causing a slight jerk.

\section{CONCLUSION}

In this paper, a model reference controller has been developed to coordinate the motor torque, engine torque, and clutch torque during mode transition for an SPHEV. The MRC takes the motor-only driving vehicle as the reference model, and the controller acts on the output errors between the reference model and vehicle as the feedback signals to achieve smooth and efficient mode transitions. A dynamic model has been developed for the MRC design, and model-based analysis has been performed to derive the stability conditions for the 
closed-loop system. For the overactuated system with three inputs and two outputs, the guidelines for selecting input combinations and feed-forward and feedback parameters have been proposed. Using the proposed control law and parameter selection method, continuous clutch torques can be achieved.

Several factors that can influence the performance of the MRC mode transition have been studied through simulations. The proposed MRC has yielded good performance for different values of feed-forward variables, which implies that the MRC is adaptive to different torque distribution strategies. The small switch trigger parameter $\varepsilon$ has been found to have significant impact on reducing frictional loss. The estimation error of vehicle load $T_{r}(t)$ and the noise of clutch actuation torque $T_{c}(t)$ introduce apparent vibrations to the vehicle, whereas the estimation error of engine resistant torque $T_{f}(t)$ and the noise of engine output torque $T_{e}(t)$ have no substantial effect on the vehicle driveline dynamics.

The MRC method is applied to the mode transition of an SPHEV bus. The simulation and experimental results confirm that the MRC outperforms the conventional operation method by reducing torque interruption for the driveline, vehicle jerk, and frictional losses. The promising results, particularly the experimental validation, have motivated us to further pursue the idea of MRC and seek its robust and effective implementation to other mode transition control problems on other hybrid vehicle platforms.

\section{ACKNOWLEDGMENT}

The authors would like to thank Prof. H. Peng and Prof. R. Singh for their valuable suggestions on this research and Dr. W. Xiong for his help in the experiments.

\section{REFERENCES}

[1] M. Ehsani, Y. Gao, S. Gay, and A. Emadi, Modern Electric, Hybrid Electric, and Fuel Cell Vehicles: Fundamentals, Theory, and Design. Boca Raton, FL: CRC Press, 2009.

[2] M. Ehsani, Y. Gao, and J. M. Miller, "Hybrid electric vehicles: Architecture and motor drives," Proc. IEEE, vol. 95, no. 4, pp. 719-728, Apr. 2007.

[3] J.-Y. Park, Y.-K. Park, and J.-H. Park, "Optimal power distribution strategy for series-Parallel hybrid electric vehicles," Proc. Inst. Mech. Eng., Part D-J. Autom. Eng., vol. 222, no. 6, pp. 989-1000, Jun. 2008.

[4] K. Koprubasi, Modeling and Control of a Hybrid-Electric Vehicle for Drivability and Fuel Economy Improvements. Columbus, $\mathrm{OH}$ : Ohio State Univ. Press, 2008.

[5] S. Kim, J.-Y. Park, J.-H. Hong, M.-W. Lee, and H.-S. Sim, "Transient control strategy of hybrid electric vehicle during mode change," presented at the SAE World Congr. Exhibition, Detroit, MI, 2009, SAE Tech. Paper 2009-01-0228.

[6] W. Lhomme, R. Trigui, P. Delarue, B. Jeanneret, A. Bouscayrol, and F. Badin, "Switched causal modeling of transmission with clutch in hybrid electric vehicles," IEEE Trans. Veh. Technol., vol. 57, no. 4, pp. 20812088, Jul. 2008.

[7] J. Liu and H. Peng, "Modeling and control of a power-split hybrid vehicle," IEEE Trans. Control Syst. Technol., vol. 16, no. 6, pp. 1242-1251, Nov. 2008

[8] Y. Cheng, R. Trigui, C. Espanet, A. Bouscayrol, and S. Cui, "Specifications and design of a PM electric variable transmission for Toyota Prius II," IEEE Trans. Veh. Technol., vol. 60, no. 9, pp. 4106-4114, Nov. 2011.

[9] Y. Kim, J. Lee, C. Jo, Y. Kim, M. Song, J. Kim, and H. Kim, "Development and control of an electric oil pump for automatic transmissionbased hybrid electric vehicle," IEEE Trans. Veh. Technol., vol. 60, no. 5, pp. 1981-1990, Jun. 2011.
[10] W. Ryu, N. Cho, I. Yoo, H. Song, and H. Kim, "Performance analysis of a CVT clutch system for a hybrid electric vehicle," Int. J. Autom. Technol., vol. 10, no. 1, pp. 115-121, Feb. 2009.

[11] L. Chen, F. Zhu, M. Zhang, Y. Huo, C. Yin, and H. Peng, "Design and analysis of an electrical variable transmission for a series-parallel hybrid electric vehicle," IEEE Trans. Veh. Technol., vol. 60, no. 5, pp. 23542363, Jun. 2011.

[12] W. Xiong, Y. Zhang, and C. Yin, "Optimal energy management for a series-parallel hybrid electric bus," Energy Convers. Manage., vol. 50, no. 7, pp. 1730-1738, Jul. 2009.

[13] C. Duan and R. Singh, "Dynamics of a 3DOF torsional system with a dry friction controlled path," J. Sound Vib., vol. 289, no. 4/5, pp. 657-688, Feb. 2006.

[14] R. Beck, F. Richert, A. Bollig, D. Abel, S. Saenger, K. Neil, T. Scholt, and K.-E. Noreikat, "Model predictive control of a parallel hybrid vehicle drivetrain," in Proc. 44th IEEE Conf. Decision Control, Eur. Control Conf., Seville, Spain, 2005, pp. 2670-2675.

[15] O. Sundstrom, P. Soltic, and L. Guzzella, "A transmission-actuated energy-management strategy," IEEE Trans. Veh. Technol., vol. 59, no. 1, pp. 84-92, Jan. 2010.

[16] H. D. Lee, S. K. Sul, H. S. Cho, and J.-M. Lee, "Advanced gear-shifting and clutching strategy for a parallel-hybrid vehicle," IEEE Ind. Appl. Mag., vol. 6, no. 6, pp. 26-32, Nov./Dec. 2000.

[17] P. A. Ioannou and J. Sun, Robust Adaptive Control. Upper Saddle River, NJ: Prentice-Hall, 1996.

[18] K. K. Shyu, M. J. Yang, Y. M. Chen, and Y.-F. Lin, "Model reference adaptive control design for a shunt active-power-filter system," IEEE Trans. Ind. Electron., vol. 55, no. 1, pp. 97-106, Jan. 2008.

[19] Y. T. Liu, K. M. Chang, and W. Z. Li, "Model reference adaptive control for a piezo-positioning system," Precision Eng., vol. 34, no. 1, pp. 62-69, 2010.

[20] L. Yacoubi, K. Ai-Haddad, L.-A. Dessaint, and F. Fnaiech, "Linear and nonlinear control techniques for a three-phase three-level NPC boost rectifier," IEEE Trans. Ind. Electron., vol. 53, no. 6, pp. 1908-1918, Dec. 2006.

[21] K. Koprubasi, E. R. Westerevelt, and G. Rizzoni, "Toward the systematic design of controllers for smooth hybrid electric vehicle mode changes," in Proc. Amer. Control Conf., New York, 2007, pp. 2985-2990.

[22] J. Zhang, L. Chen, and G. Xi, "System dynamic modelling and adaptive optimal control for automatic clutch engagement of vehicles," Proc. Inst. Mech. Eng., Part D-J. Autom. Eng., vol. 216, no. 12, pp. 983-991, Dec. 2002.

[23] A. Crowther, N. Zhang, D. K. Liu, and J. K. Jeyakumaran, "Analysis and simulation of clutch engagement judder and stick-slip in automotive powertrain systems," Proc. Inst. Mech. Eng., Part D-J. Autom. Eng., vol. 218, no. 12, pp. 1427-1446, Dec. 2004.

[24] J. Y. Wong, Theory of Ground Vehicles. New York: Wiley-Interscience, 2001.

[25] R. L. Williams and D. A. Lawrence, Linear State-Space Control Systems. New York: Wiley, 2007.

[26] L. Chen, G. Xi, and C. Yin, "Model referenced adaptive control to compensate slip-stick transition during clutch engagement," Int. J. Autom. Technol., vol. 12, pp. 913-920, 2011.

[27] F. Amato, Robust Control of Linear Systems Subject to Uncertain TimeVarying Parameters. Berlin, Germany: Springer-Verlag, 2006.

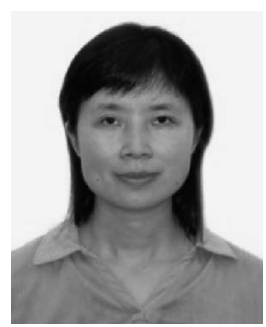

Li Chen received the B.S. and M.S. degrees in mechanical engineering from Hunan Univeristy, Changsha, China, in 1994 and 1997, respectively, and the Ph.D. degree in vehicle engineering from Shanghai Jiao Tong University, Shanghai, China, in 2000 .

She is currently an Associate Professor with the School of Mechanical Engineering, Shanghai Jiao Tong University. Her research interests include dynamics and control for advanced powertrain systems. 


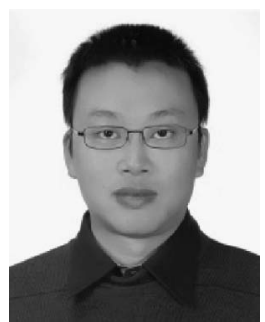

Gang Xi received the B.S. and M.S. degrees in mechanical engineering from Hunan Univeristy, Changsha, China, in 1993 and 1996, respectively, and the Ph.D. degree in vehicle engineering from Shanghai Jiao Tong University, Shanghai, China, in 2002.

$\mathrm{He}$ is currently a Senior System Engineer with the United Automotive Electronic Systems Company, Ltd., Shanghai. His research interests include high-efficiency technologies in vehicle powertrain systems.

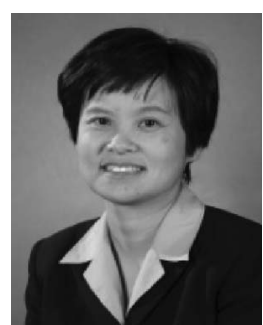

Jing Sun (F'04) received the B.S. and M.S. degrees from the University of Science and Technology of China, Hefei, China, in 1982 and 1984, respectively, and the Ph.D. degree from the University of Southern California, Los Angeles, in 1989.

From 1989 to 1993, she was an Assistant Professor with the Department of Electrical and Computer Engineering, Wayne State University, Detroit, MI. She joined Ford Research Laboratory in 1993, where she worked in the Powertrain Control Systems Department. After spending almost ten years in the industry, she came back to academia and joined the Faculty of the College of Engineering, University of Michigan, Ann Arbor, in 2003. She is currently a Professor with the Department of Naval Architecture and Marine Engineering, University of Michigan. She is the holder of 37 U.S. patents. He is a coauthor of a textbook on robust adaptive control. Her research interests include system and control theory and its applications to marine and automotive propulsion systems.

Dr. Sun is one of the three recipients of the 2003 IEEE Control System Technology Award. 